\title{
InfiniCloud: Leveraging the Global InfiniCortex Fabric and OpenStack Cloud for Borderless High Performance Computing of Genomic Data and Beyond
}

\author{
Kenneth Ban \\ Medicine, National University of Singapore \\ Address, Singapore \\ E-mail: fkbaneimcb.a-star.edu.sg \\ Jakub Chrzeszczyk \\ National Computational Infrastructure (NCI) \\ Australian National University, Australia \\ E-mail: jakub. chrzeszczykeanu.edu.au
}

Institute of Molecular and Cell Biology, A*STAR, Dept. of Biochemistry, Yong Loo Lin School of

\section{Andrew Howard}

National Computational Infrastructure (NCI)

Australian National University, Australia

E-mail: andrew.howardeanu.edu.au

\section{Dongyang Li}

National Computational Infrastructure (NCI)

Australian National University, Australia

E-mail: dongyang. Iieanu.edu.au

\section{Tin Wee Tan}

$A * S T A R$ Computational Resource Centre $\left(A^{*} C R C\right)$, Dept. of Biochemistry, Yong Loo Lin School of Medicine, National University of Singapore

Address, Singapore

E-mail: tinweedbic.nus.edu.sg

At the Supercomputing Frontiers Conference in Singapore in 2015, A*CRC (Singapore) and NCI (Canberra, Australia) successfully presented InfiniCloud, a geographically distributed, high performance InfiniBand HPC Cloud which aims to enable borderless processing of genomic data as part of the InfiniCortex project. This paper provides a high-level technical overview of the architecture of InfiniCloud and how it can be used for high performance computation of genomic data in geographically distant sites by encapsulation of workflows/applications in Virtual Machines (VM) coupled with on-the-fly configuration of clusters and high speed transfer of data via long range Infiniband.

International Symposium on Grids and Clouds (ISGC) 2015

15 -20 March 2015,

Academia Sinica, Taipei, Taiwan 


\section{Introduction}

The advent of big data has driven the need for flexible high performance computing platforms in order to analyze large amounts of data using user defined workflows, particularly in the emerging field of genomics and healthcare informatics. These workflows typically require a specific stack of applications with their Operating System-specific dependencies, which can be different for each pipeline and can frequently change over time as updates are released. In addition to the heterogenous nature of applications, such workflows demand high CPU performance paired with large memory capability as well as a high-performance interconnect for analysis of large genomic/healthcare datasets.

In response to this growing need for high performance and flexible computing for analysis of large datasets, $\mathrm{A} * \mathrm{CRC}$ and $\mathrm{NCI}$ teams collaborated to define a new cloud computing platform called InfiniCloud, which combines high performance (HPC) cloud computing powered by OpenStack with the high speed/low latency Infiniband network architecture. This platform was optimized to provide high performance computing with minimal overhead within virtual instances, coupled with native Infiniband protocol to provide high speed interconnect and transfer of data between the instances.

We have extended the InfiniCloud platform to address another major challenge in the computation of large datasets such as genomic data: the need for efficient high speed transfers to and from the HPC cloud computing platform. To do this, we leveraged the long range Obsidian Longbow E100 Infiniband extenders to enable unprecedented high speed transfer of large datasets and VM images across trans-Pacific distances between two geographically distant InfiniCloud platforms in Singapore and Canberra via Seattle. This capability enables borderless high performance cloud computing by high speed transfer of large datasets together with workflows/applications encapsulated in VMs. Furthermore workflows/applications in VMs can be parallelized in virtual instances by the on-the-fly setup of cluster compute nodes.

We envision that the InfiniCloud platform combined with long range Infiniband as part of a global fabric (InfiniCortex) will enable seamless distributed high performance computing amongst geographically distant InfiniCloud nodes, breaking down borders on the path to exascale computing to meet the challenge of big data computing.

\section{InfiniCloud Platform}

The InfiniCloud platform was developed on compute cluster hardware at the NCI and A*CRC based on OpenStack cloud computing software stack with custom modifications. 


\subsection{Hardware Components:}

Currently, InfiniCloud consists of two sites: one located at the NCI (National Computational Infrastructure), in Canberra, Australia (Figure 1) and the second at $\mathrm{A}^{*} \mathrm{CRC}$, Singapore (Figure 2). The total count of compute cores available is 264, supporting 3TB of memory and a local storage capacity of $15 \mathrm{~TB}$ (SSD and HDD). All instances are connected to the shared 56Gbit FDR IB fabric.

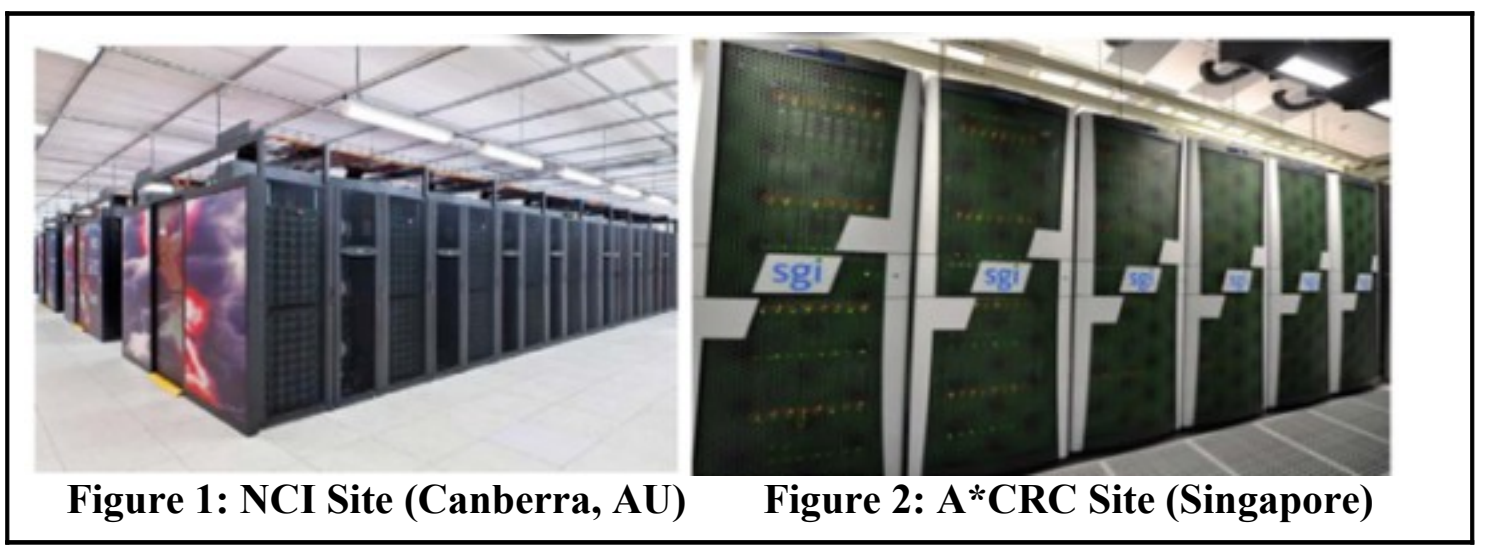

\subsubsection{Server Specifications}

The overall design of each site is similar, utilizing a common InfiniBand interconnect. The server configurations are detailed in Tables 1 and 2.

Table 1. NCI Compute Cluster Hardware Configuration

\begin{tabular}{|l|l|}
\hline Servers & 10x Fujitsu PRIMERGY CX250 machines \\
\hline CPU & Intel Xeon E5-2650 \\
\hline Memory & 256GB or 128GB \\
\hline Interconnect & FDR IB \\
\hline Local storage & $4 \mathrm{x}$ Intel DCS3700 or 6x 2.5 inch 10k SAS \\
\hline
\end{tabular}

Table 2. A*CRC Compute Cluster Configuration:

\begin{tabular}{|l|l|}
\hline Servers & $5 \mathrm{x}$ SGI C1104-GP1 \\
\hline CPU & Xeon E5-2680 \\
\hline Memory & $128 \mathrm{~GB}$ \\
\hline Interconnect & FDR IB \\
\hline Local storage & $1 \mathrm{x}$ Intel DCS3500 or 3x Intel DCS3500 \\
\hline
\end{tabular}

\subsubsection{Local Area Network Components (each site):}


Table 3: Network Configuration

\begin{tabular}{|l|l|}
\hline Switching & FDR IB \\
\hline Range extender & Obsidian Strategics Longbow E100 \\
\hline Subnet manager & OpenSM (active: AU; standby: SG) \\
\hline
\end{tabular}

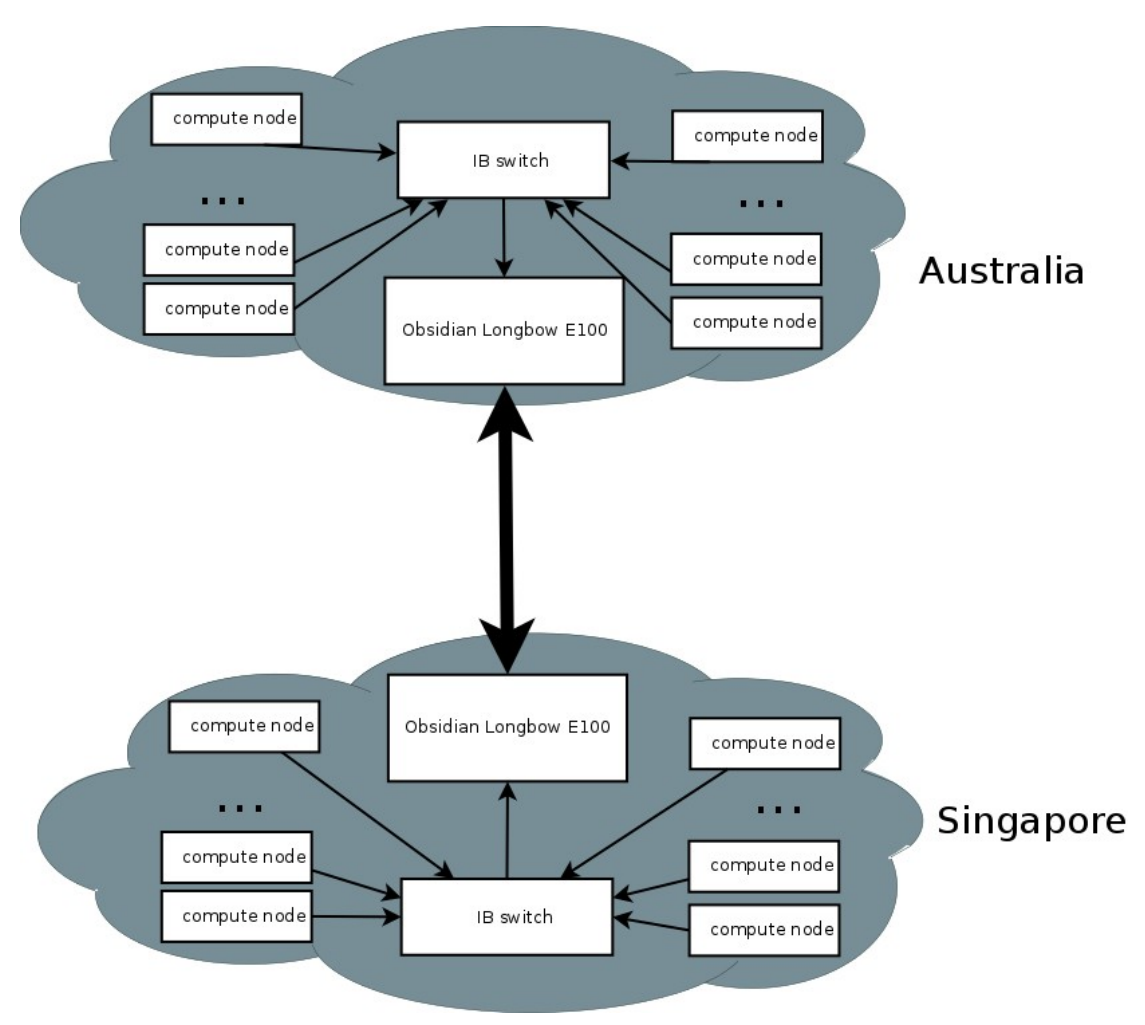

Figure 3: InfiniCloud Network Topology

\subsubsection{Global Area Network:}

To enable the global InfiniBand connection, the A*CRC and NCI teams worked closely with AARNet (AU), SingAREN (SG) and Pacific NorthWest GigaPop (PNWGP) in Seattle (USA) to secure a dedicated 10Gbit/s layer 2 link between Canberra and Singapore (Figure 4). Due to the network topology connecting Australia (with the majority of the bandwidth provided to the more densely populated East Coast of Australia), the link was routed via the longer eastern path, crossing the Pacific Ocean twice through PNWGP in Seattle with an RTT of $305 \mathrm{~ms}$. In contrast, the more direct western path through Western Australia, Indian Ocean and Guam while exhibiting better delay characteristics has limited capacity and is only capable of providing a $1 \mathrm{Gbit} / \mathrm{s}$ connection. 


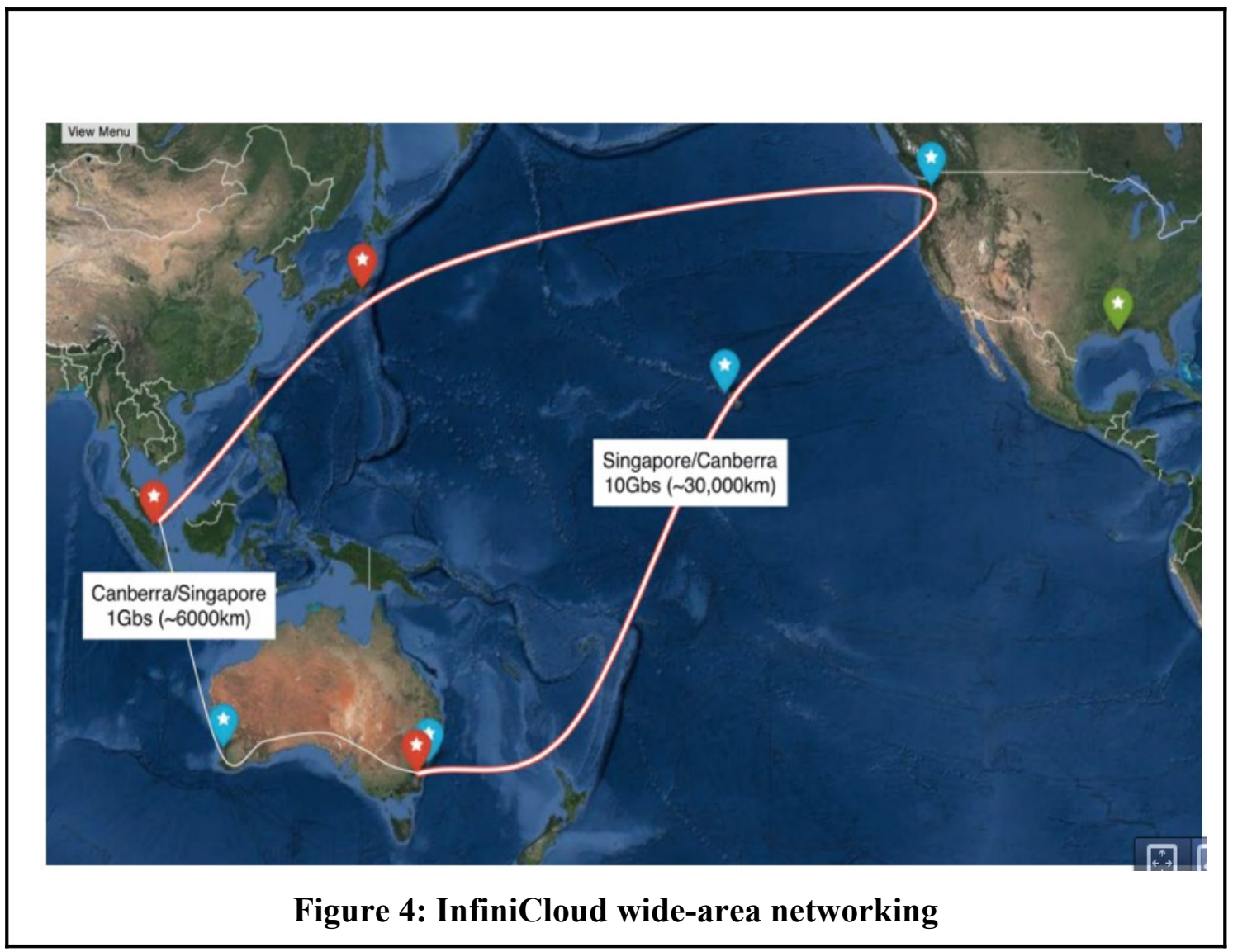

\subsection{InfiniCloud Installation and Configuration}

All InfiniCloud systems run the following software stack (Table 4):

Table 4: InfiniCloud Software Stack

\begin{tabular}{|l|l|}
\hline Operating System & CentOS 6.6 x86_64 \\
\hline InfiniBand drivers & Mellanox OFED 2.4 \\
\hline OpenStack version & Icehouse + InfiniCloud specific patches \\
\hline
\end{tabular}

Both InfiniCloud clusters consist of one dedicated management node, one dedicated controller node and a variable number of compute nodes (ranging from 4-8). All node classes are integrated to form a fully featured HPC Cloud.

- The management node is used for bare metal provisioning and cluster-wide configurations.

- The controller node provides API, CLI and GUI access to the Cloud and is responsible for managing all the core areas of cluster operation: identity management, scheduling, VM image storage, network management and providing an orchestration layer. 
- Compute nodes provide CPU, RAM, storage and high performance SRIOV networking to the virtual instances.

Building the InfiniCloud cluster required a high degree of customization in order to enable native InfiniBand capability in virtual instances, as well as to provide access to the global InfiniBand network connecting Australia and Singapore. The diagram and table below (Table 5; Figure 5) show the components that were modified to enable full native Infiniband capabilities:

Table 5: OpenStack Customizations

\begin{tabular}{|l|l|l|}
\hline Neutron Server & enable SRIOV and native IB capability & Controller side \\
\hline Neutron Networker & enable EoIPoIB support & Networker side \\
\hline Nova Compute & enable SRIOV and native IB capability & Compute side \\
\hline Neutron Agent & enable SRIOV and native IB capability & Compute side \\
\hline DHCP & enable IPoIB support & Networker side \\
\hline eswitchd & customize Pkey assignments to create global IB fabric & Networker side \\
\hline
\end{tabular}

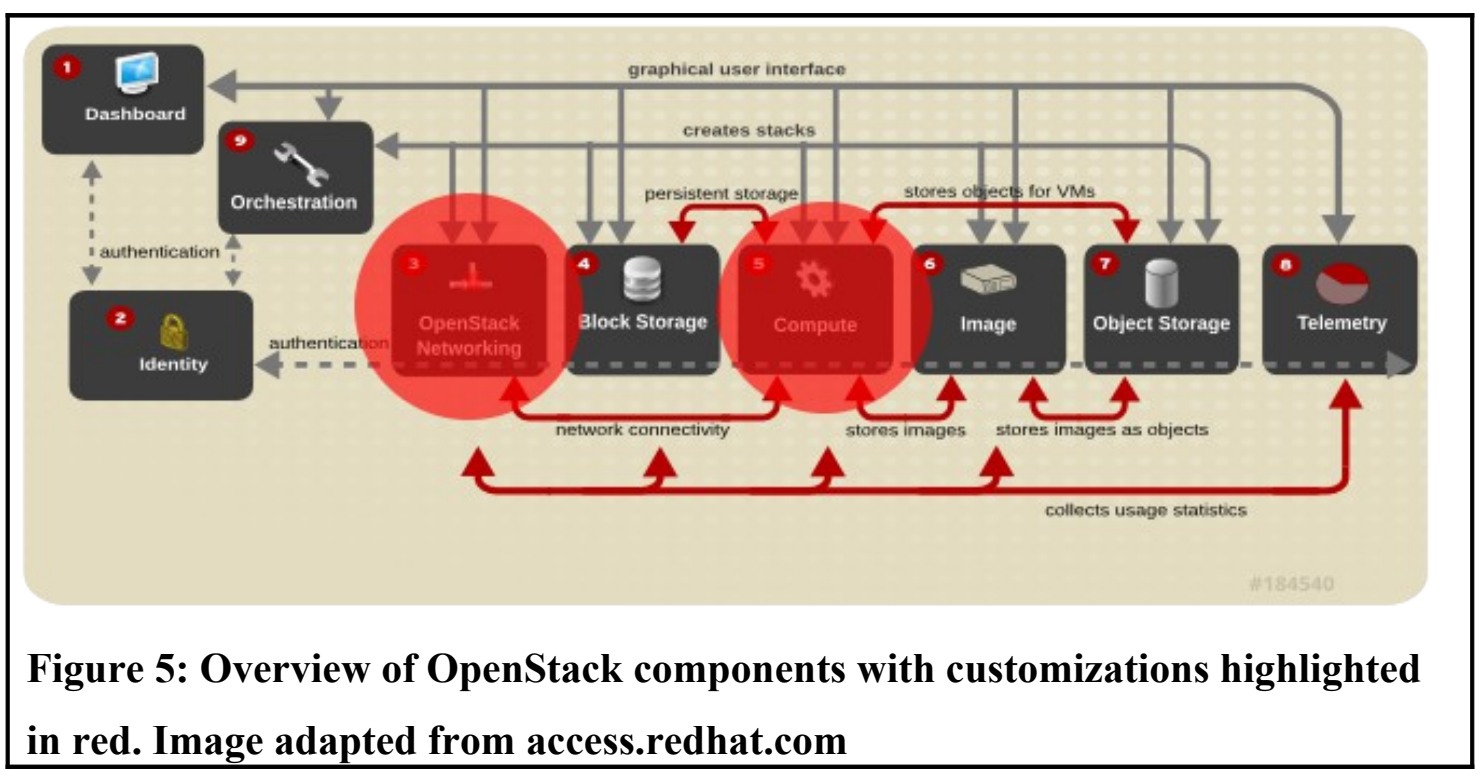

\section{InfiniCloud Infiniband Capabilities}

After cloud provisioning is complete and all the customizations required for global InfiniBand communications are in place, the system has the ability to provide virtual instances on demand, connected over InfiniBand, with full ability to communicate to remote instances using RDMA over a trans-Pacific 10Gbit/s network. 


\subsection{Native IB capability in VM:}

Infiniband IP assignments for 2 virtual OpenStack instances (NFS-DATA-AU and NFSREF-AU) in Infinicloud:

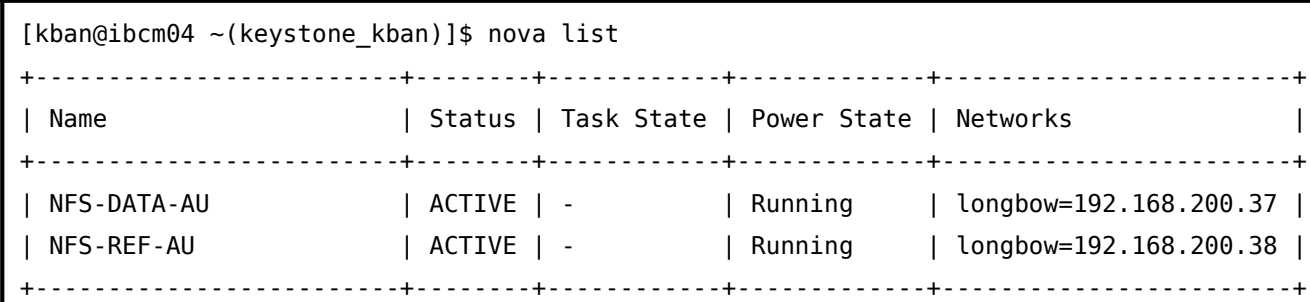

IP configuration in one of the virtual instances (NFS-REF-AU):

[kban@ibcm04 $\sim$ (keystone_kban)]\$ ssh 192.168.200.38

[ root@nfs-ref-au ] \# ifconfig

Ifconfig uses the ioctl access method to get the full address information, which limits hardware addresses to 8 bytes.

Because Infiniband address has 20 bytes, only the first 8 bytes are displayed correctly.

Ifconfig is obsolete! For replacement check ip.

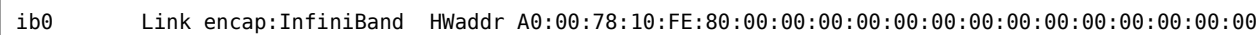

inet addr:192.168.200.38 Bcast:192.168.200.255 Mask:255.255.255.0

inet6 addr: fe80::fa16:3e00:a3:6dfe/64 Scope:Link

UP BROADCAST RUNNING MULTICAST MTU: 2044 Metric: 1

RX packets: 65071 errors: 0 dropped:0 overruns: $\theta$ frame: 0

TX packets: 13377 errors: $\theta$ dropped: 0 overruns: $\theta$ carrier: $\theta$

collisions: 0 txqueuelen: 1024

RX bytes:33891502 (32.3 MiB) TX bytes:2237704 (2.1 MiB)

\subsection{Native IB capability in VM:}

We demonstrate high bandwidth capability $(\sim 6 \mathrm{~GB} / \mathrm{sec})$ between 2 virtual instances:

\begin{tabular}{|c|c|c|c|c|}
\hline \multicolumn{5}{|c|}{ RDMA_Write BW Test } \\
\hline Dual-port & : OFF & Device & : $\mathrm{mlx} 4 \_0$ & \\
\hline Number of qps & $: 1$ & Transport type & : IB & \\
\hline \multirow{2}{*}{\multicolumn{2}{|c|}{ 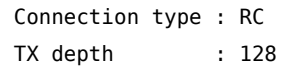 }} & Using SRQ & : OFF & \\
\hline & & & & \\
\hline \multicolumn{2}{|c|}{ CQ Moderation : 100} & & & \\
\hline \multicolumn{5}{|c|}{ Mtu $\quad: 2048[B]$} \\
\hline \multicolumn{5}{|c|}{ Link type $\quad$ : IB } \\
\hline \multicolumn{5}{|c|}{ Max inline data : $\theta[B]$} \\
\hline \multirow{2}{*}{\multicolumn{5}{|c|}{$\begin{array}{ll}\text { rdma_cm QPs } & : \text { OFF } \\
\text { Data ex. method : Ethernet }\end{array}$}} \\
\hline & & & & \\
\hline \multicolumn{5}{|c|}{$\begin{array}{l}\text { local address: LID } 0 \times 05 \text { QPN } 0 \times 0 a 5 e \text { PSN } 0 \times 90 c 425 \text { RKey } 0 \times b 8011700 \text { VAddr } 0 \times 007 f 476 c 430000 \\
\text { remote address: LID } 0 \times 1 a \text { QPN } 0 \times 0 \text { cac PSN 0x94503d RKey 0x7001182b VAddr } 0 \times 007 f a 941670000\end{array}$} \\
\hline \multicolumn{5}{|c|}{ \#bytes $\quad$ \#iterations $\quad$ BW peak $[\mathrm{MB} / \mathrm{sec}] \quad$ BW average $[\mathrm{MB} / \mathrm{sec}] \quad$ MsgRate[Mpps] } \\
\hline \multicolumn{5}{|c|}{$\begin{array}{lll}65536 & 5000 & 5984.52\end{array}$} \\
\hline
\end{tabular}




\subsection{High (10Gbit) bandwidth capability - global:}

Integral to the data transfer component is the use of the Obsidian Strategics DSYNC utility which utilises the RDMA (Remote Direct Memory Access) capabilities to provide long range Infiniband RDMA transfers between Infiniband-connected virtual instances.

Here we demonstrate the high speed transfer of $381 \mathrm{~GB}$ of genomic data in under 9 minutes from the $A^{*} \mathrm{CRC}$ Infinicloud (Singapore) to NCI (Canberra, Australia) via the $10 \mathrm{G}$ link going through Seattle $(\sim 30,000 \mathrm{~km})$ using the DSYNC utility.

[root@testo1 ] \# dsync --direct-io - option Xfer::RDMA::Buffer-Size=5368709120 --option Xfer: : RDMA : : IO-Block-Size=10485760 192.168.200.144:/scratch/kuba/reference_dset/ /scratch/kubatest/

Finished generating remote file list. 40 files, 3 directores, 381GB.

Finished checking local files. Need to get 40 files, 381GB.

Transfer xfer-ib-rdma network usage 3050B in $0 \mathrm{~s}$ (10.0kB/s)

Transfer xfer-ib-rdma network usage $381 \mathrm{~GB}$ in $8 \mathrm{~m} 19 \mathrm{~s}$ (764MB/s)

Done. Transferred 381GB in $8 \mathrm{~m} 27 \mathrm{~s}$ (752MB/s)

The remarkable performance observed with long range Infiniband RDMA provides a significant improvement over standard TCP/IP protocols.

\section{Using InfiniCloud for Parallelized Workflows in Genomic Analysis}

The InfiniCloud platform provides a high performance cloud computing environment for flexible workflows, coupled with unprecedented high speed transfer of big data sets over large geographical distances. A key application that takes advantage of these high performance characteristics is the analysis of genomic sequences which has seen an exponential growth in demand with the advent of next generation sequencing technologies. 


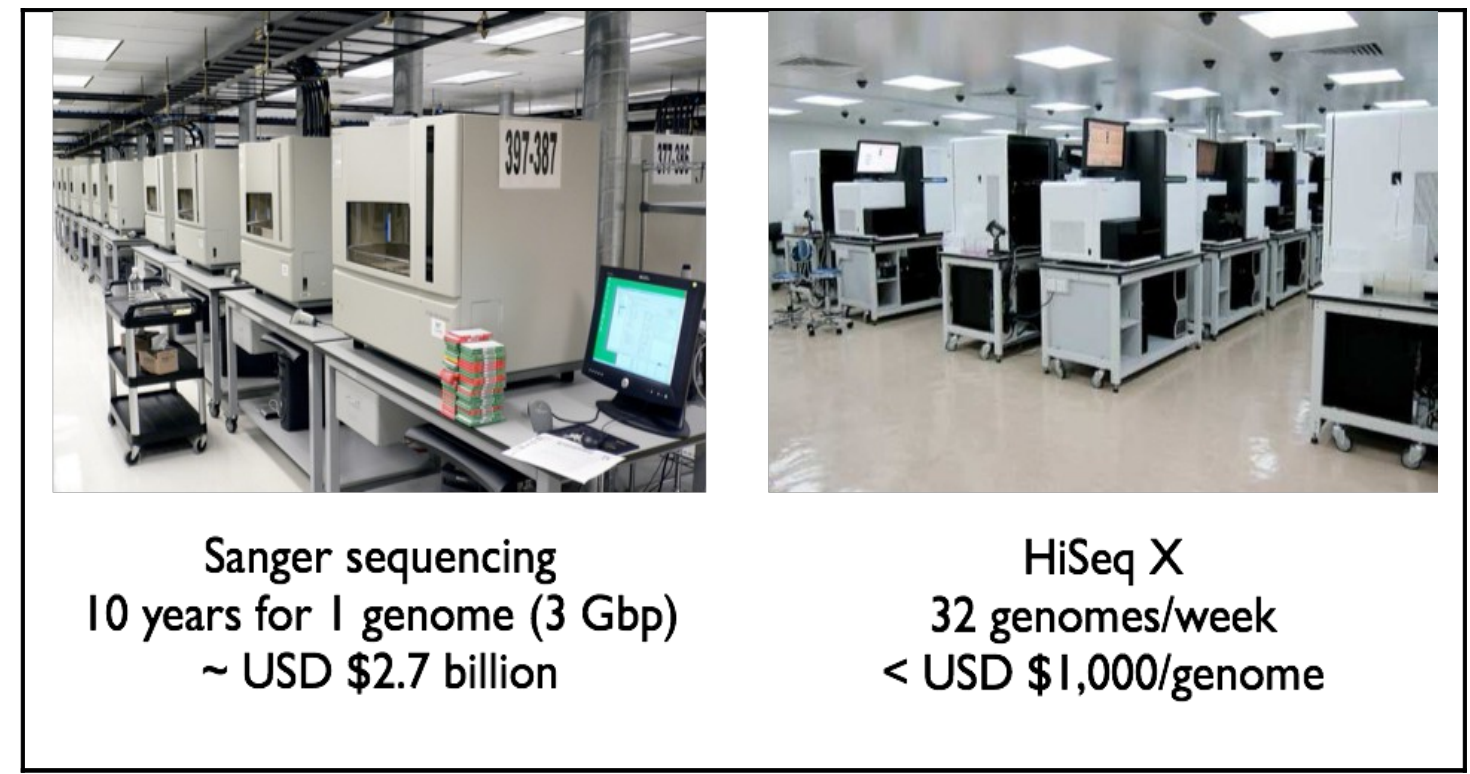

The rapid development of next generation of sequencing technologies has dramatically reduced the cost of sequencing genomes. Previously, it took $\sim$ USD $\$ 2.7$ billion and 10 years to sequence one human genome, but currently the cost has dropped several orders of magnitude to $\sim$ USD $\$ 1,000$ per genome with the introduction of platforms such as the Illumina HiSeq X sequencer. This drop in cost coupled with the ability to sequence a complete human genome in a few days has driven the adoption of genomic sequencing in research labs as well as hospitals.

Although the cost and speed of sequencing has dramatically improved, the transfer and computation of the genomic data remains a bottleneck in translating that data into the insights needed for improving patient care. In addition, the computational analysis of genomic data for clinical use requires enforcement of reproducibility standards in addition to the data provenance and security guarantees needed to comply with ethical and legal privacy issues. A computational platform for clinical genomics needs to meet the following challenges:

- High speed data transfers from sequencing data stores to the computational platform

- Reproducible and well documented workflows that can be run on different hardware platforms

- Easy provisioning of compute clusters for processing genomic data from multiple samples using parallel workflows

- High CPU and network performance for rapid analysis of large datasets

- Mechanisms for data provenance and security (e.g. using ephemeral containers) for computation at remote sites

4.1 Provisioning of instances and on-the-fly setup of cluster compute nodes for parallel workflows 
To address these challenges, we implemented a software stack on top of the InfiniCloud platform that leverages the use of VM instances or containers to encapsulate workflows, together with automated provisioning of virtual instances and the setup of virtual compute clusters for parallelized workflows.

We adapted Elasticluster (http://gc3-uzh-ch.github.io/elasticluster/) for use on InfiniCloud to enable easy provisioning of instances and setup of virtual clusters for parallel workflows (Figure 6). In our custom configuration, Elasticluster was used to provision instances and set up a virtual cluster consisting of a frontend node and a user-defined number of compute nodes. To enable cluster computing for parallel workflows, we configured Elasticluster to install and setup the SGE job scheduler, Ganglia monitoring tools, and the IPython notebook shell (Table 6).

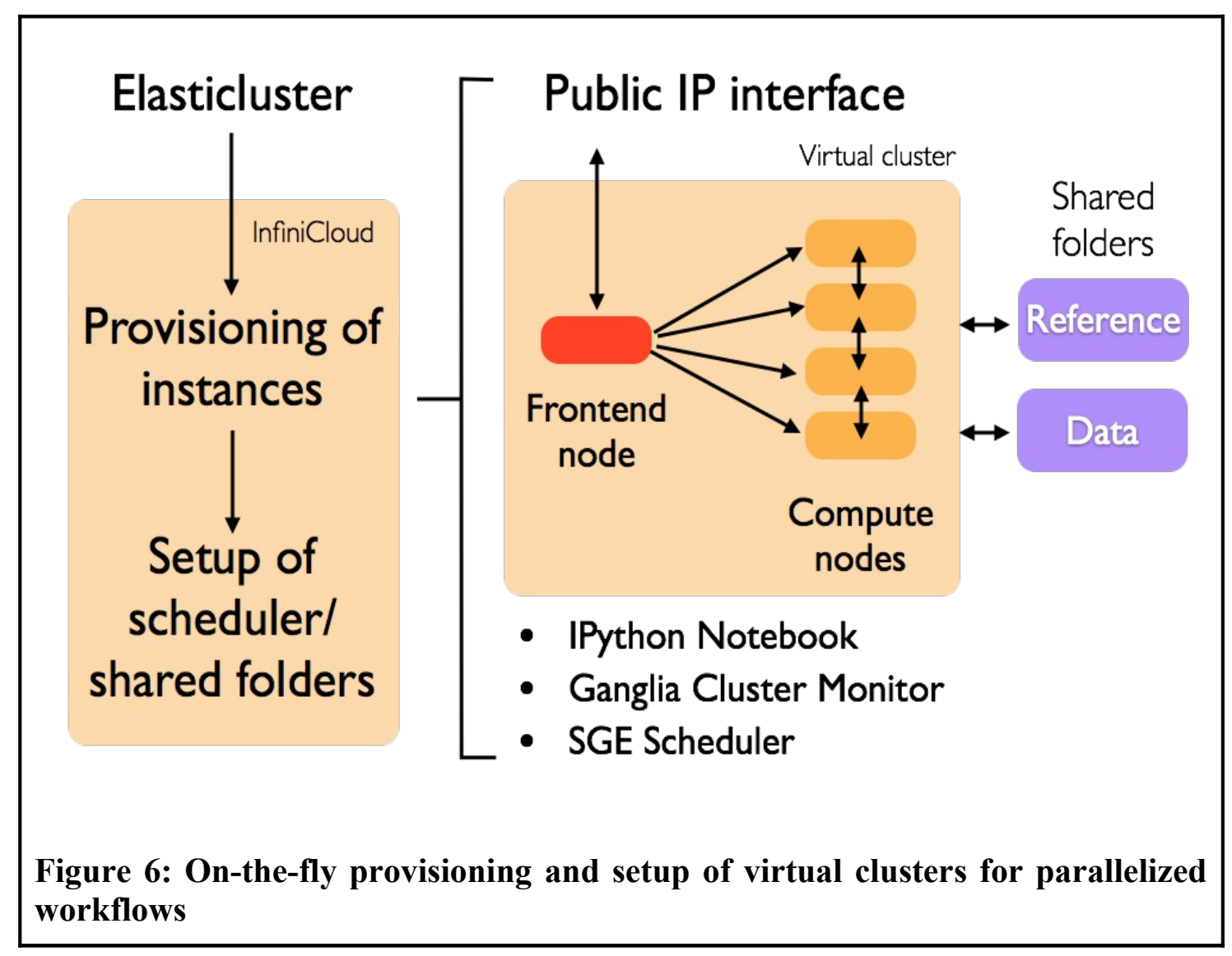


Table 6: Cluster computing stack

\begin{tabular}{|l|l|l|}
\hline IPython & $\begin{array}{l}\text { Notebook shell (BASH/Python/R) } \\
\text { IPython parallel engine }\end{array}$ & 2.4 .1 \\
\hline SGE & Grid engine job scheduler & $6.2 \mathrm{u} 5$ \\
\hline Ganglia & Cluster monitoring (CPU/Memory/Network) & 3.1 .7 \\
\hline
\end{tabular}

We demonstrate the ease of provisioning and setup of a virtual compute cluster on InfiniCloud using Elasticluster:

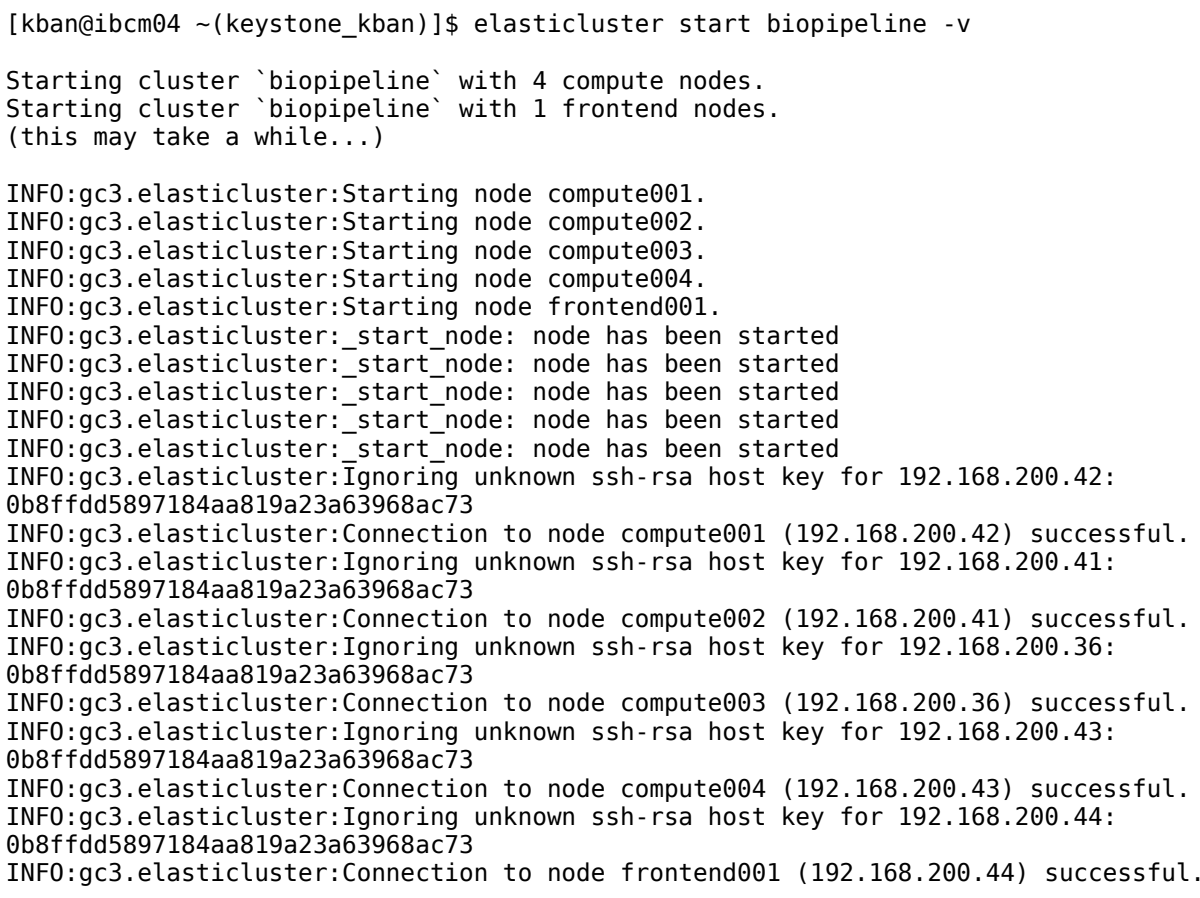

Following provisioning of virtual instances, the setup of virtual cluster is done automatically using Ansible Playbooks as a backend (abbreviated output):

Configuring the cluster.

(this too may take a while...)

\section{Setup of SSH for all nodes}

$\ldots$

TASK: [Setup SSH host based authentication (server configuration file; enable ‘HostbasedAuthentication`option)] ***

changed: [frontend001]

changed: [compute002]

changed: [compute004]

changed: [compute001]

changed: [compute003] 


\section{Setup of Ganglia Monitor}

TASK: [Install ganglia monitor (Cent0S)]

changed: [ compute002] $\Rightarrow>$ (item=ganglia-gmond)

changed: [ compute004] $\Rightarrow$ (item=ganglia-gmond)

changed: [ compute001] $\Rightarrow$ (item=ganglia-gmond)

changed: [ compute003] $\Rightarrow$ (item=ganglia-gmond)

...

\section{Setup of SGE Job Scheduler}

TASK: [Install GridEngine RPM packages]

changed: [ frontend001] => (item=gridengine-qmaster,gridengine-execd,gridengine-qmon) ...

\section{Setup IPython Notebook Shell Web Interface}

PLAY [IPython controller Playbook (standard user operations)]

TASK: [create ipython profile]

changed: [compute001]

changed: [compute 002 ]

changed: [compute004]

changed: [compute003]

changed: [frontend 001 ]

$\ldots$

At completion of setup, the user is able to access the frontend node via SSH:

INF0: gc3.elasticluster:Cluster correctly configured.

Your cluster is ready!

Cluster name: biopipeline

Frontend node: frontend001

- compute nodes: 4

- frontend nodes: 1

To login on the frontend node, run the command:

elasticluster ssh biopipeline

To upload or download files to the cluster, use the command:

elasticluster sftp biopipeline

Control of cluster and job submission through frontend node using the SGE scheduler:

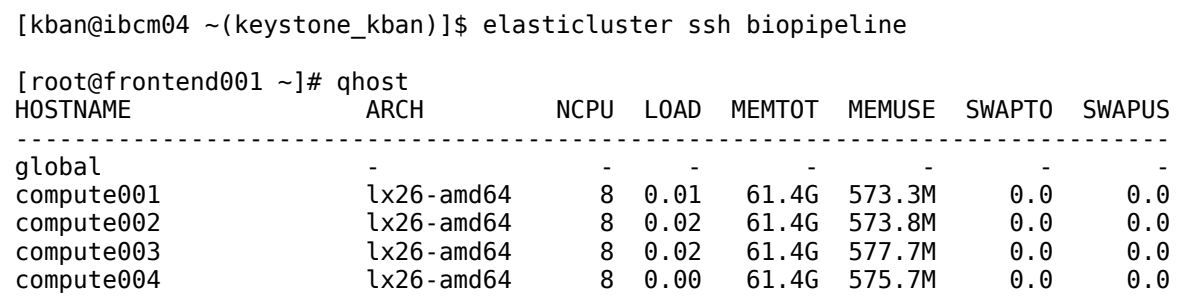


Besides SSH access, the setup provides a web interface for cluster monitoring using Ganglia:

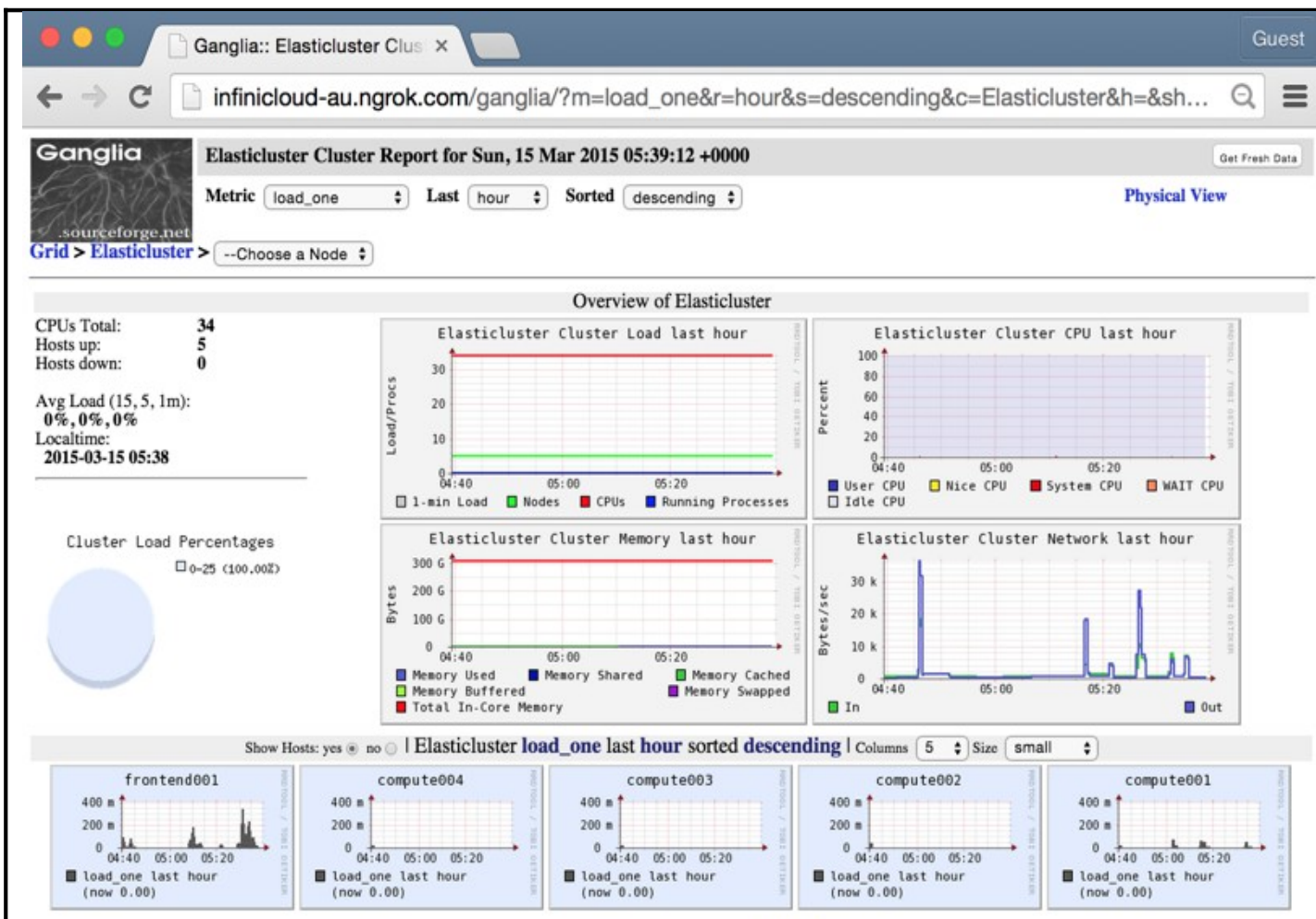

(Nodes colored by 1-minute load) I Legend

Finally, the setup provides a versatile IPython Web Notebook interface for BASH/Python/R scripting:

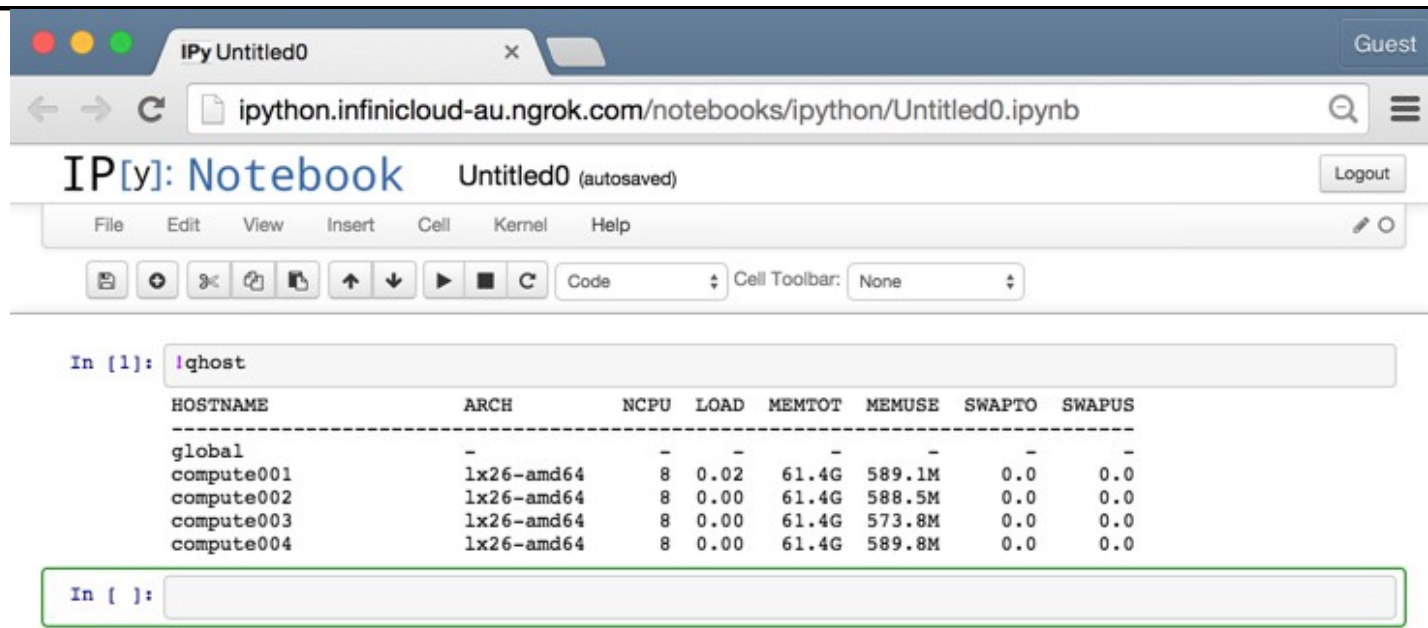




\subsection{Implementation of variant calling genome analysis pipeline}

Next, we demonstrate how the on-the-fly provisioning and setup of a virtual machine which can be used to parallelize a genomic analysis workflow. We chose a clinically relevant workflow, called variant calling, that takes genomic sequences from cancer samples and detects mutations in genes that could be used to determine the prognosis of a patient, or to identify potential chemotherapy drugs that could be used for treatment. Because each cancer sample can be analyzed separately, the workflow is amenable to simple asynchronous parallelization without any interprocess communication.

In this workflow, genomic sequences are processed in a pipeline through a series of steps using different applications to identify and annotate mutations (Figure 7). We use a pipeline application to orchestrate the steps in processing and to distribution the processing to the compute nodes using the SGE scheduler.

1. Genomic sequences from each cancer sample are processed with an aligner - an application that compares the sequences to a human reference genome sequence and identifies the position and alignment of each sequence from the cancer samples.

2. The files from each cancer sample are processed by a variant caller program, which compares the aligned sequences to the human reference genome sequence to identify variations (substitutions, insertions, deletions) in the cancer samples.

3. The variant files from each cancer sample are annotated. A specialized application compares each variation to multiple databases to identify what the potential effects of each mutation have on regions in the genome.

The applications are pre-installed in the VM images together with their dependencies to enable portability between InfiniCloud platforms. The reference datasets required by the aligner, variant caller, and annotation tool, are located in a data volume that can be mirrored between InfiniCloud platforms. The genomic dataset is isolated in a separate volume which also stores the results of the analysis (Figure 7); this isolation provides the flexibility for maintaining data provenance and security 


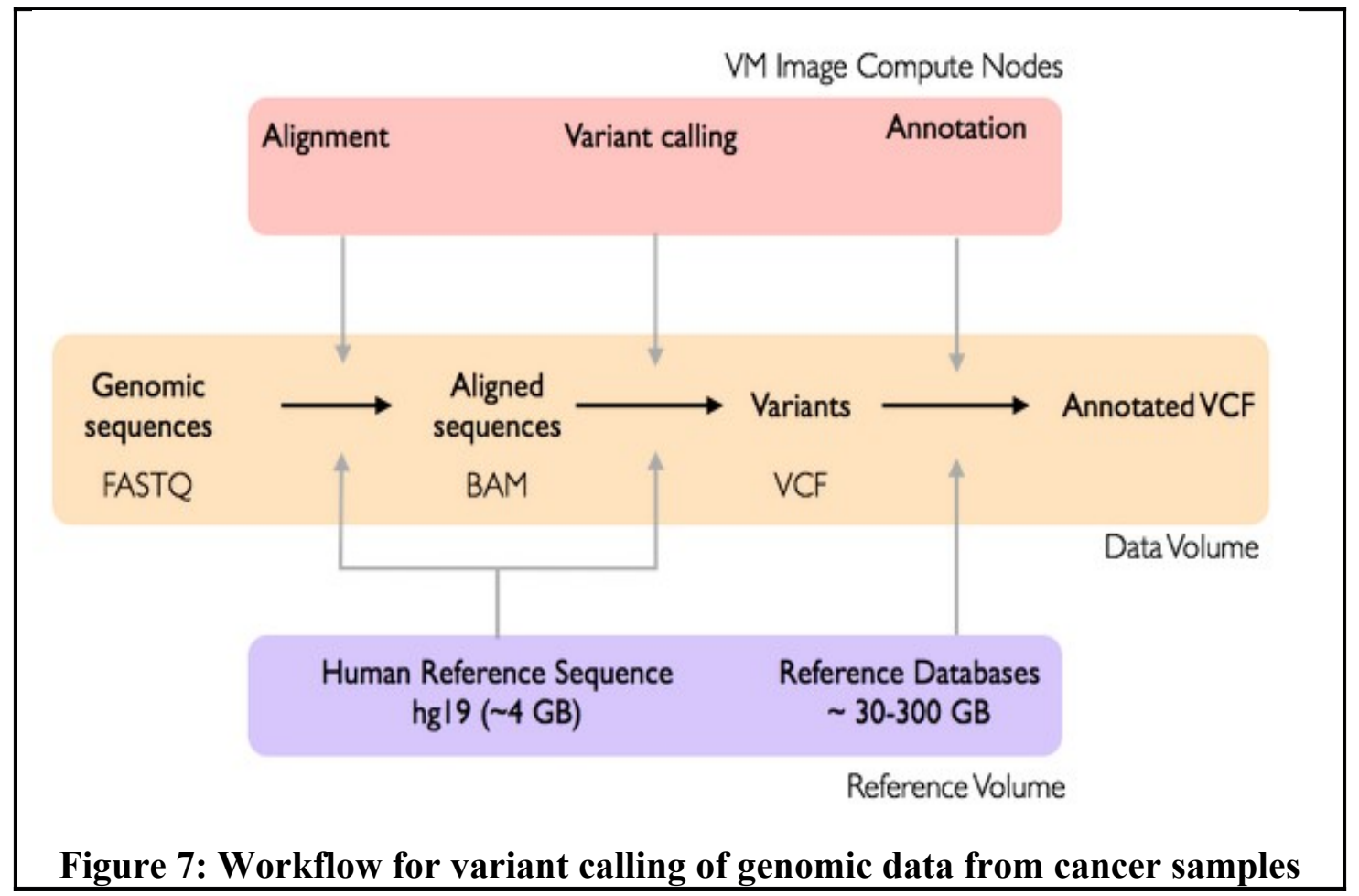

\subsection{Demonstration of genome analysis workflow for remote cloud computing}

We demonstrate the computation of genomic sequences on the InfiniCloud platform in Canberra, Australia from Singapore by remote provisioning of instances, setup of the cluster, and mounting of reference/data volumes (Figure 8):

- The VM images are mirrored from Singapore to Australia so that both sites have the same application/workflow backends for genomic analysis

- The common reference volume is automatically mirrored from Singapore to Australia and attached to the frontend and compute nodes

- The data volume is synchronized according to a user-defined workflow and attached to the frontend and compute nodes

- Genomic data is transferred from Singapore to Australia for computation

- Results are transferred back from Australia to Singapore

- Data volume on remote site can be deleted in cases where genomic data cannot be stored offsite for data provenance and security reasons 


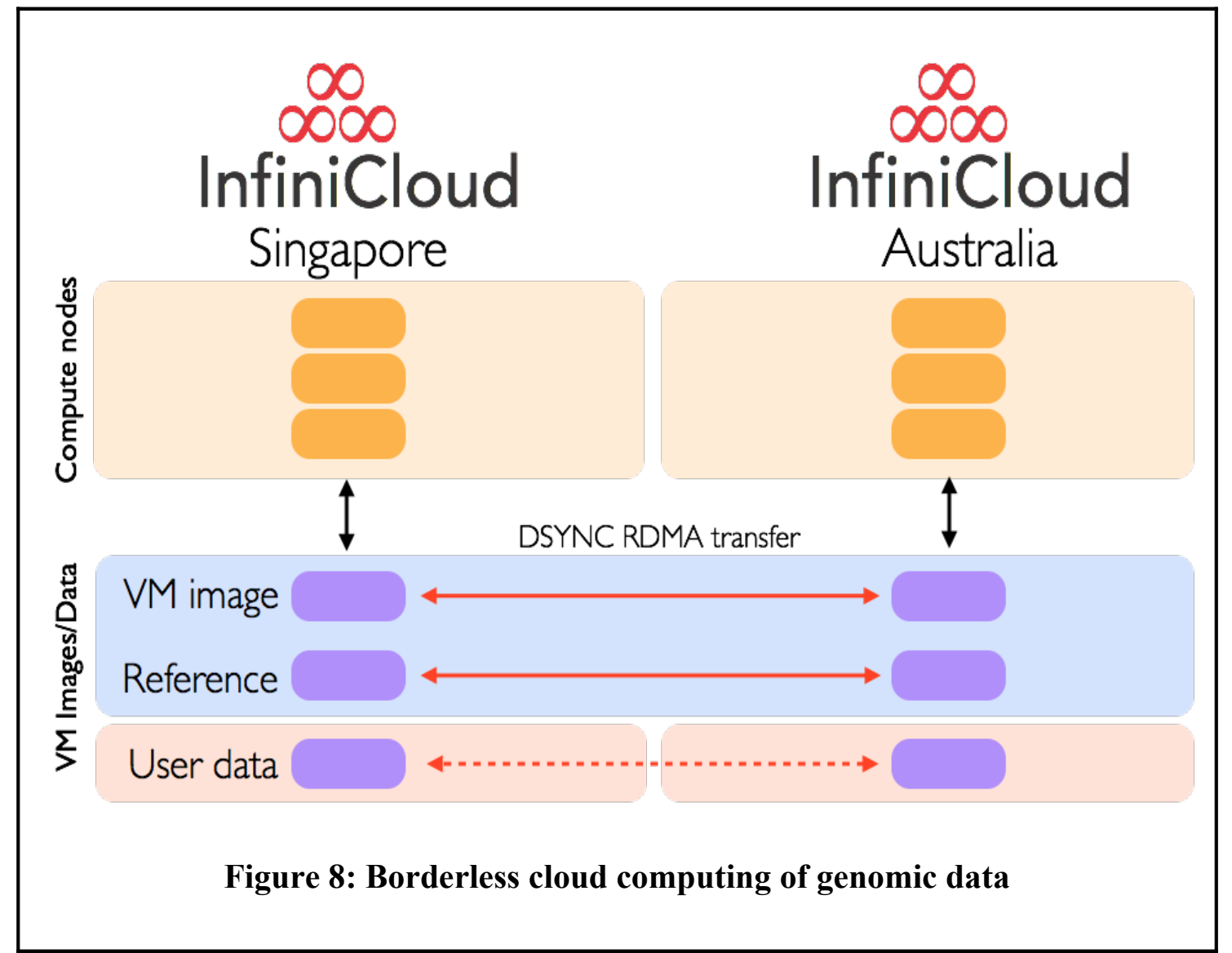

For the analysis, the genomic data is transferred from Singapore into the data volume (Australia) using dsync. Here, we achieve a transfer of $\sim 233$ GB of data in 5.5 minutes ( 696 $\mathrm{MB} / \mathrm{sec})$ from Singapore to Australia via Seattle $(\sim 30,000 \mathrm{~km})$ :

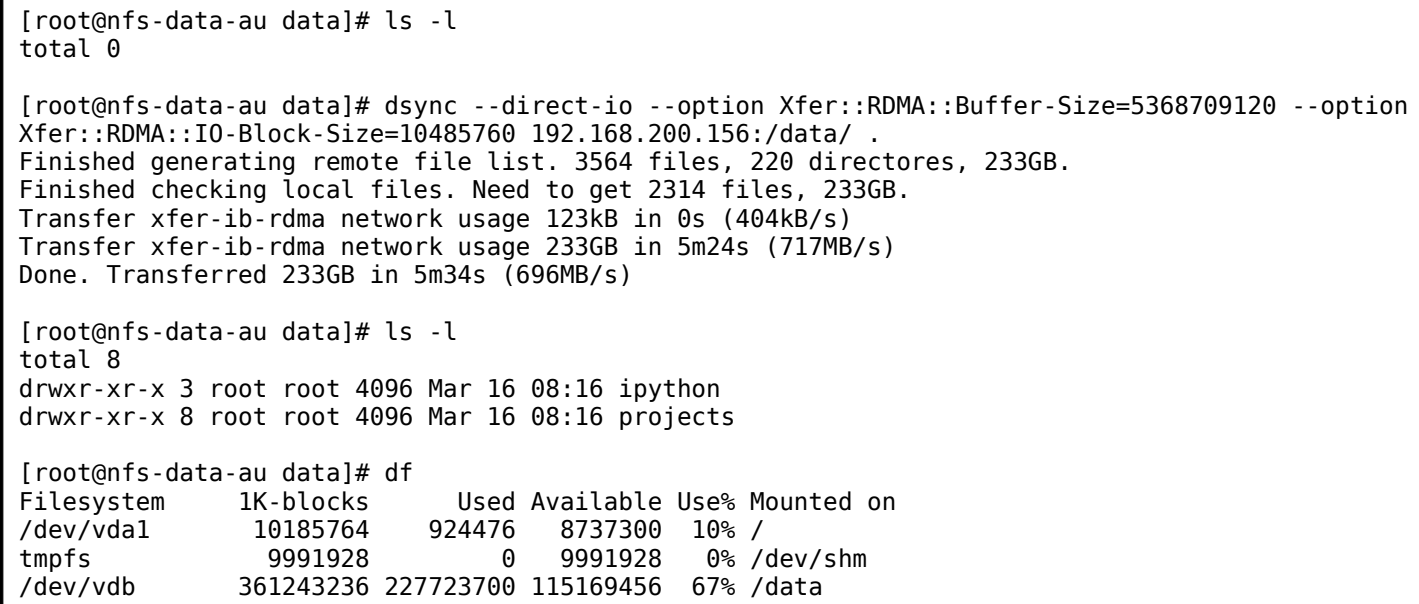


Here we run the pipeline remotely on InfiniCloud (Australia) for variant calling on genomic sequences from cancer samples using bpipe, which interprets the workflow script specified in the variant_calling.pipe file. The abbreviated output is shown below:

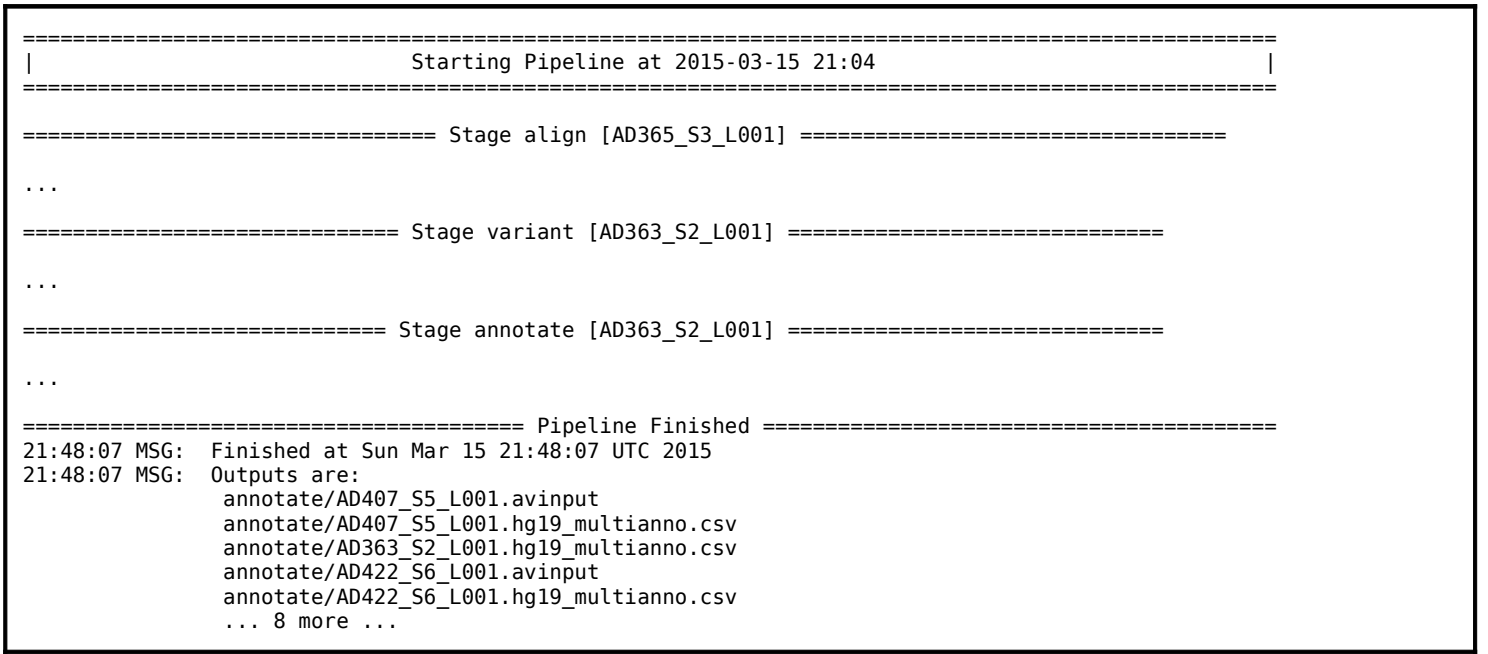

Ganglia monitoring of the workflow showing aggregate CPU and network loads on all compute nodes:

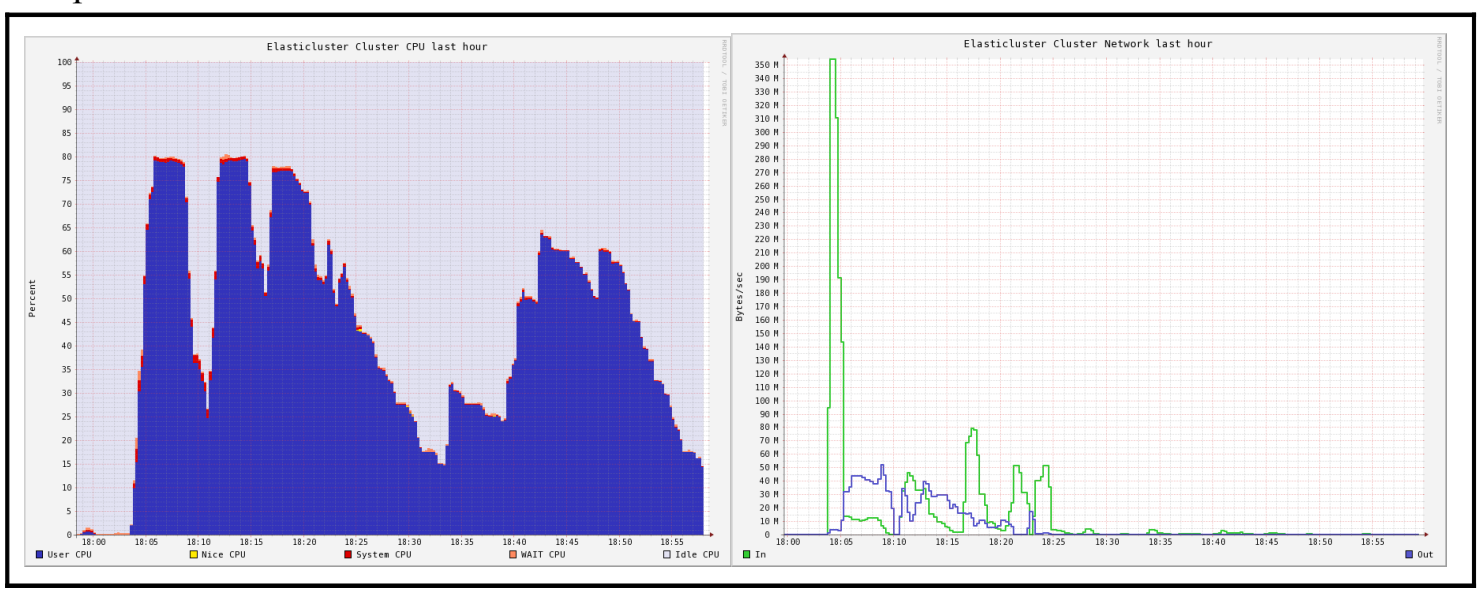

Example of mutations detected in a tumour suppressor gene (TP53) in a cancer sample which generally signifies a bad prognosis:

\begin{tabular}{|c|c|c|c|c|c|c|c|c|}
\hline Chr & Start & End & Ref & Alt & Func.refGene & \multicolumn{2}{|c|}{ Gene.refGen ExonicFunc.refGene } & snp138 \\
\hline chr17 & 7574025 & 7574025 & C & - & exonic & TP53 & frameshift deletion & . \\
\hline chr17 & 7577531 & 7577531 & G & - & exonic & TP53 & frameshift deletion & . \\
\hline chr17 & 7579472 & 7579472 & G & C & exonic & TP53 & nonsynonymous SNV & rs1042522 \\
\hline chr17 & 7579644 & 7579659 & CCCCAGCCCTCCAGGT & - & intronic & TP53 & . & rs 146534833 \\
\hline chr17 & 7579801 & 7579801 & G & C & UTR5 & TP53 & . & rs1642785 \\
\hline
\end{tabular}


Upon completion of the analysis, the results in the data volume are transferred back to the point of origin (Singapore) and the volume is deleted. This workflow provides one approach to maintain data provenance at the site of origin while harnessing the high performance computational resources at remote sites.

\section{Conclusions}

We present a new cloud computing platform called InfiniCloud, which combines High Performance Cloud Computing powered by OpenStack with the high speed/low latency of an Infiniband network architecture. This platform delivers High Performance Computing with minimal overhead within virtual instances, coupled with native Infiniband protocol for high speed interconnect transfer of data between the instances.

The InfiniCloud platform incorporates long range Infiniband extension and enables unprecedented high speed transfers of large datasets and VM images across global distances. The $\mathrm{A}^{*}$ Star and NCI teams have successfully demonstrated data transfer across trans-Pacific distances $(\sim 30,000 \mathrm{~km})$ between two geographically distant InfiniCloud platforms located in Singapore and Canberra, Australia. This capability enables borderless high performance cloud computing supporting high speed transfer of large datasets together with workflows/applications encapsulated in Virtual Machines. This encapsulation allows parallelization of virtual instances and on-the-fly instantiation of cluster compute nodes using Elasticluster.

We envision that the InfiniCloud platform combined with long range Infiniband as part of the InfiniCortex global InfiniBand fabric, will enable seamless distributed cloud based High Performance Computing amongst geographically distant InfiniCloud nodes, breaking down borders and illuminating the path to Exascale computing to meet the challenge of supporting todays and tomorrows big data computing needs. 